\title{
Focaltheorem - A portfolio Web Application
}

\author{
Vishesh Kumar Sharma ${ }^{a}$, Stephen Arnold K ${ }^{b}$, Prabhat Mishrac ${ }^{c}$ Mr. Himanshu Sharma ${ }^{d}$ \\ a,b,c Department of Computer Science, Galgotias University, Greater Noida, UP India \\ ${ }^{\mathrm{d}}$ Guide, Assistant Professor, Galgotias University, Greater Noida,UP \\ avishukaushik1998@gmail.com, ${ }^{\mathrm{b}}$ stephenarnoldno1@gmail.com, ${ }^{\mathrm{c}}$ princumishrad@gmail.com, \\ dhimanshusharma@galgotiasuniversity.edu.in
}

Article History: Received: 10 November 2020; Revised 12 January 2021 Accepted: 27 January 2021; Published online: 5 April 2021

\begin{abstract}
This project is used to upload photos and videos easily through one platform. We deployed this in cloud so it is very easy to use and accessible to others. In this we use the technology of web development. So this portfolio is very helpful for photographers for uploading any photographs. Platform gives clients free, limitless capacity for photographs up to 16 megapixels and recordings up to 1080p goal. The help consequently breaks down photographs, distinguishing different visual highlights and subjects. Clients can look for anything in photographs, with the assistance returning outcomes from three significant classifications: People, Places, and Things. Stage perceives faces, gathering comparative ones (this component is just accessible in specific places because of protection laws), geographic tourist spots, and subject matter, including college events in Galgotias University, Uni-Fests, Club Events, F1 Race, Food-Fest, and more.
\end{abstract}

Keywords:

\section{Introduction}

Focaltheorem is a photograph imparting stage to Scalable capacity administration created by group of three individuals. Various types of Services in web application permits acknowledgment of photograph substance, naturally produce collections, energize comparable photographs into brisk recordings, surface past recollections at critical occasions, and improve the nature of photographs and recordings. Portfolio have approximately a few updates in Future, including updates for and recommended sharing of photographs, divided photograph libraries among two clients, and actual collections, with Photos naturally proposing assortments dependent on face, area, trip, or other differentiation. Focaltheorem portfolio assists with keeping Back-up, where photographs can be sent or in Google terms 'Shared' without any problem. This is only a typical reinforcement apparatus when photographs are divided among web-based media or different stages or applications. Commentators preferred the refreshed Photos administration for its acknowledgment innovation, search, applications, and stacking times. The help has site supporting for the Andriod and IOS Devices of any screen size. Clients back up their photographs to the cloud administration, which become open for the entirety of their gadgets. The Photos administration breaks down and arranges pictures into gatherings and can distinguish highlights.

From the application's hunt window, clients are shown expected looks for gatherings of photographs in three significant classifications: People, Places, and Things. The help dissects photographs for comparable faces and gatherings them together in the People class. It can likewise

follow faces as they age. The Places classification utilizes information yet can likewise decide areas in more seasoned pictures by dissecting for significant tourist spots.

\section{Literature Review}

Our project is used to upload photos and videos easily through one platform. We Hosted it on InfinityFree Web Hosting so it is very easy to use and accessible to others.In this we have used the technology of web development.

InfinityFree provides Free web-Hosting with Unlimited Bandwidth, Unlimited Disk Space, and NO forced ads with $100 \%$ uptime and various options with database. So it helps our project with full accessibility and availability and ease of use for our users and for us as well. This makes it cost effective along with reducing the workload of maintaining the hosting cost and storage problems.

We have designed the main page and navigationthat helps to move from one page to another for fulfill the given requirements of the user.We basically created all pages responsive as well as attractive. 


\section{Description of Modules and Implementation}

We implemented this project using web development.We hosted it on InfinityFree. In it we store the photos as per events and time. We use PHP code that fetch photos from folder stored on Hosting platform storage then display them in designed format.

Different categories of photos are given in navigation bar where we can move into that category page and look the photos of particular event.

In it we created and implemented different web pages say homepage,contact us,about us and then different sub pages for different categories.

\section{A. Tools Used for Implementation}

For Frontend:

- HTML used to build the structure of pages and to give headings, paragraphs and links.

- CSS used to describe the presentation of web-pages including layout, colors, and fonts.

- BOOTSTRAP used for designing the web pages.

- JQUERY adds more features to web pages.

For Backend:

- PHP is used to fetch the photos from folder stored on hosted platform storage.

- JS used on both client side and server side that allows you to make web page interactive and more responsive.

- ajax used in backend of page.

\section{B. Roles and Responsibilities}

We founded that for in any event two reasons, that it is imperative to convey unmistakably to understudies the parts of the different people associated with a final year project. Initially, the regularizing type of assessment we use can confound the understudy's perspective on the parts of Guide and Users.

Besides, numerous activities depend on modern cooperation, where the understudy frequently has a contact individual at the accomplice organization. For this situation, the jobs of the modern contact individual and the director should be clarified. This is to evade issues emerging from misled assumptions and errors about the different obligations of individuals included.

To impart the different jobs plainly to the understudies, we use drawings like the one in Figure 1 which delineate the parts of - and connections between - the different entertainers. Along with the drawings, we portray the parts as follows: Developers (front-end and Back-end), Industrial contact individual, Users and Guide.

\section{Front-End Developer}

Front-End Developer is the driving force in the project, has responsibility to structure the web page and designing \& styling the fonts, layout and colors, adding graphics and animation, making the page responsive and more user friendly and interactive. The Front-end developer also has the responsibility of continuously presenting the current status of the project, and thereby make it possible for the supervisor to provide feedback.

\section{Back-End Developer}

Back-End Developer is responsible for working of website and make its active and provide it more features. It has responsibility to add photos and fetch them from the Host Storage and connect the website with DBMS and make it secure and live to users. Any problems that arise during the project must be brought to the Developer's attention by the guide through the users, so that the guide is given a fair chance to provide advice. The Developer is also responsible for making the necessary contacts with industrial partners, as well as for making a detailed timeplan for the project.

\section{The Guide or Supervisor}

The supervisor should provide feedback andadvice to the student. It is important to realize that since the student is expected to be the driving force in the project, the amount of help and guidance received from the supervisor depends largely on how well the student informs the supervisor on the current status of the project. The supervisor is encouraged to meet each student once per week at a fixed time, and requiring the student to send an updated version of the project report one or two days prior to each meeting. If exceptional problems turn up in a project, it is 
the responsibility of the supervisor to notify the examiner that things are not going well, so that examiner and supervisor can discuss what action should be taken. Supervisors for these research-oriented projects include primarily faculty members and Ph.D. students within the subject area.

\section{The Industrial Contact Person}

If the project is done with an industrial partner, it is fundamental to understand that the contact-individual at the organization isn't director for the undertaking. The job of the contact-individual is to give data about the issue that should be addressed, to depict requests on the arrangement that the organization looks for, and so on. In the event that the issue is too specific for the specific organization to be of any broad interest, it is the duty of the understudy to change the difficult detailing so that it fulfills the needs positioned on the difficult definition in the final year project. In the event that the issue cannot be summed up in that manner, the understudy ought to pick another issue or another modern accomplice. On the whole matters concerning the report composing, the understudy should look for counsel firstly from the director and not from the industrial contact-person.

\section{The Users}

Users plays an important role in using the web app and provides the feedback according to performance of the web app and helps in making it more accurate and bugs fee. This project is built for the users to provide them photos and pictures of the events take place in college campus. This is a platform for users to have access to all the photos and allow users to share, like and download the photos.

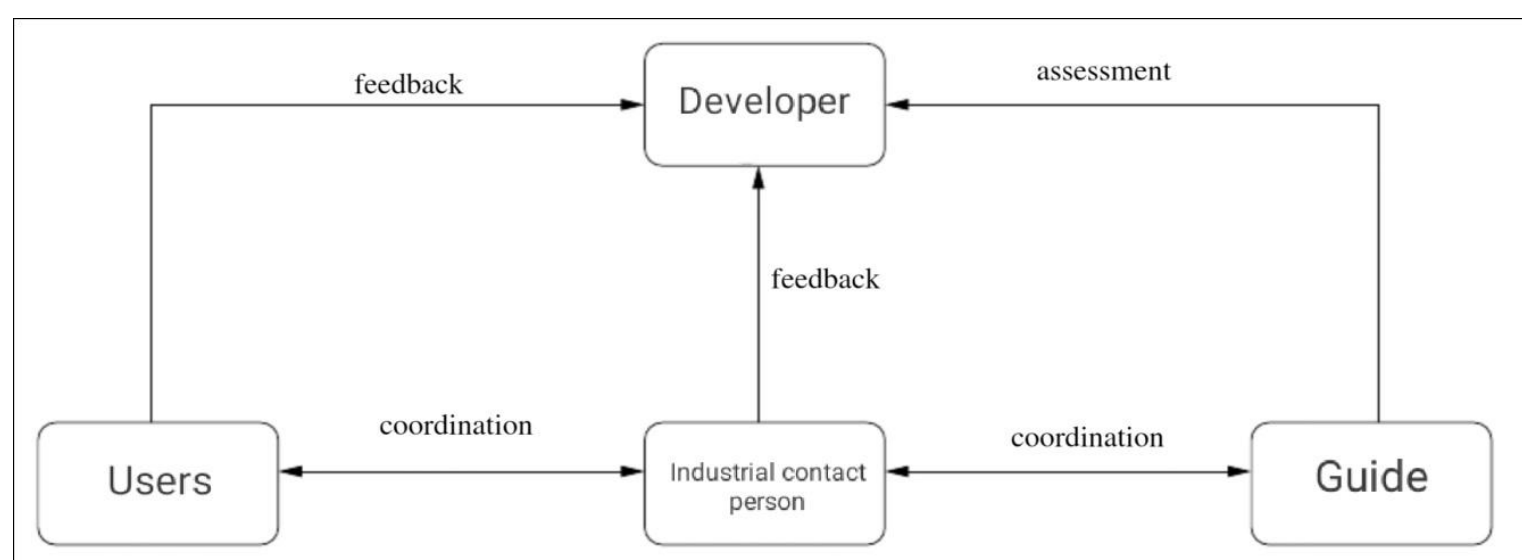

Figure 1: Relations between the main actors involved in a final year project.

\section{The assessment}

At a fixed cutoff time towards the finish of the course, understudies are needed to deliver a total draft of their report. Duplicates of each report are disseminated to the manager/guide, and two individual understudies. Every understudy should give an oral 20-minute introduction of their undertaking, trailed by 20 minutes of inquiries from the two individual understudies who have perused the report. The understudy is allowed an extra week to set up the final version.

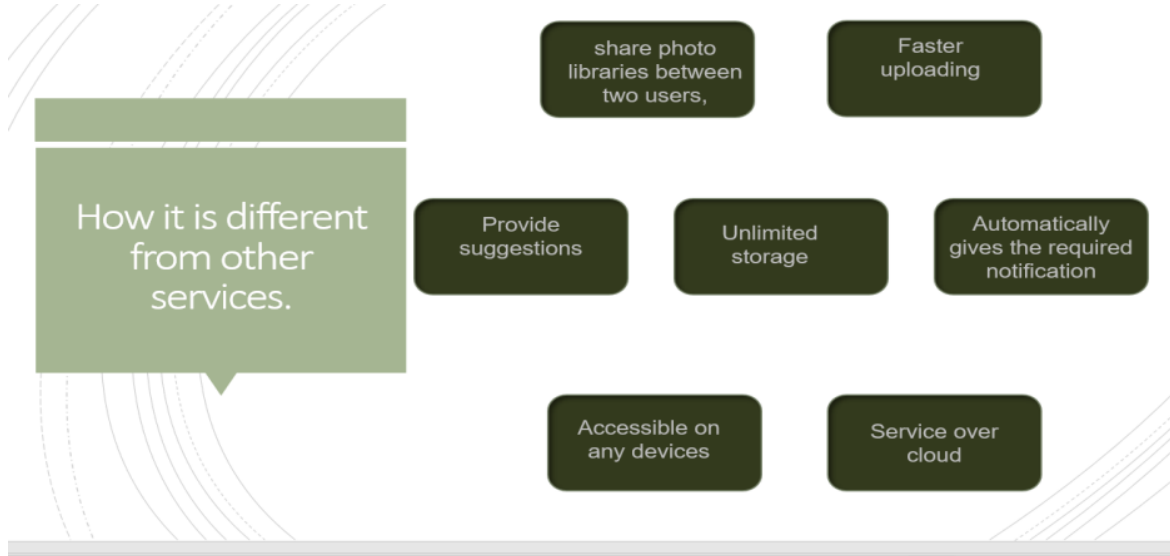

Figure 2: How it is Different 


\section{Merits or Advantages}

A. Students can access all the photos or videos of any event in campus on single platform.

B. Easy to access and responsive \& user friendly.

C. Scalable storage to store any no. Of photos and videos.

D. Extra features to like, share and download photos in original resolution.

E. Easy to find and search for photos of random events as every photo is ordered and sectioned according to event time.

F. Authors and Affiliations

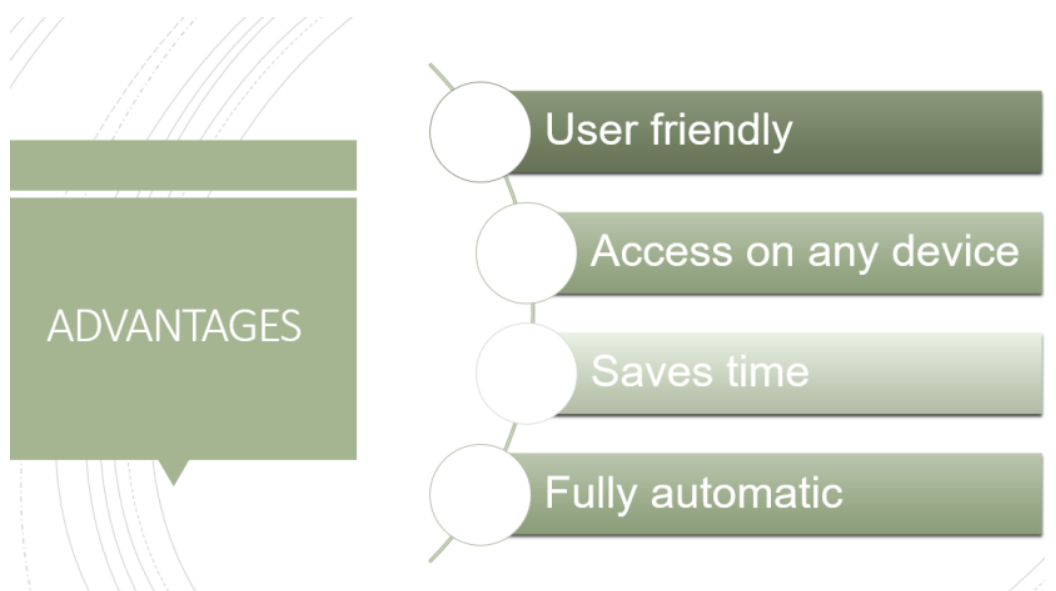

Figure 3: It's Advantages

\section{Discussion}

The main aim of this final year project is to provide a platform to the Event management group of the college to upload their photos, videos and stories of all the events they have organized, and as to share the best memories of these events with everyone so hat they can rejoice those golden moments and relive those memories of college.

It also helped us to have the experience of working in a team and a working environment as of a company. This final year project have principally detailed two positive effects. In the first place, we are more ready for the work life as issue solvers, since they know about an interaction for methodicallly taking care of issues utilizing point, destinations, and techniques. By applying this cycle, understudies that have begun to work will in general take care of issues in a more precise and complete manner (and frequently faster) than understudies that have not been presented to a comparative interaction. Having worked along these lines, organizations can allow understudies to take on more qualified and testing assignments prior in their vocations. This obviously affects the understudy's progression and self-improvement in a positive manner.

The second certain effect detailed by organizations concerns, the understudy's capacity to compose and introduce reports. Every understudy composes a report of around 40-60 pages that expounds on what the issue is, the reason it is an issue, the issue's hypothetical setting, different methodologies for how it very well may be settled, how it was tackled, how it was investigated, and ends that can be drawn. Having composed and introduced such a report additionally makes it simpler for understudies to comprehend instructions to compose and introduce great venture reports inside a organization. 


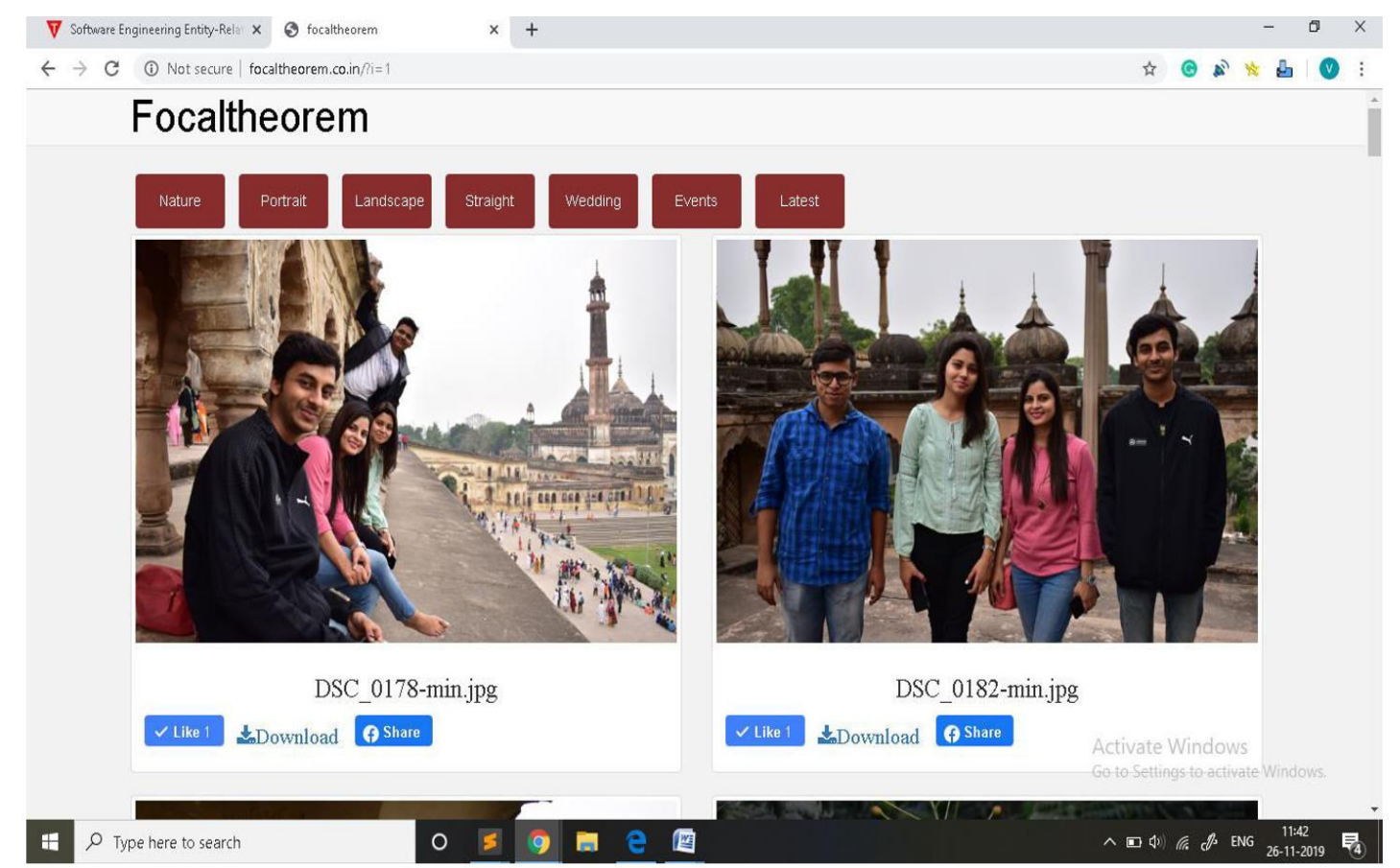

Figure 4: Home-Page of the Portfolio Gallery

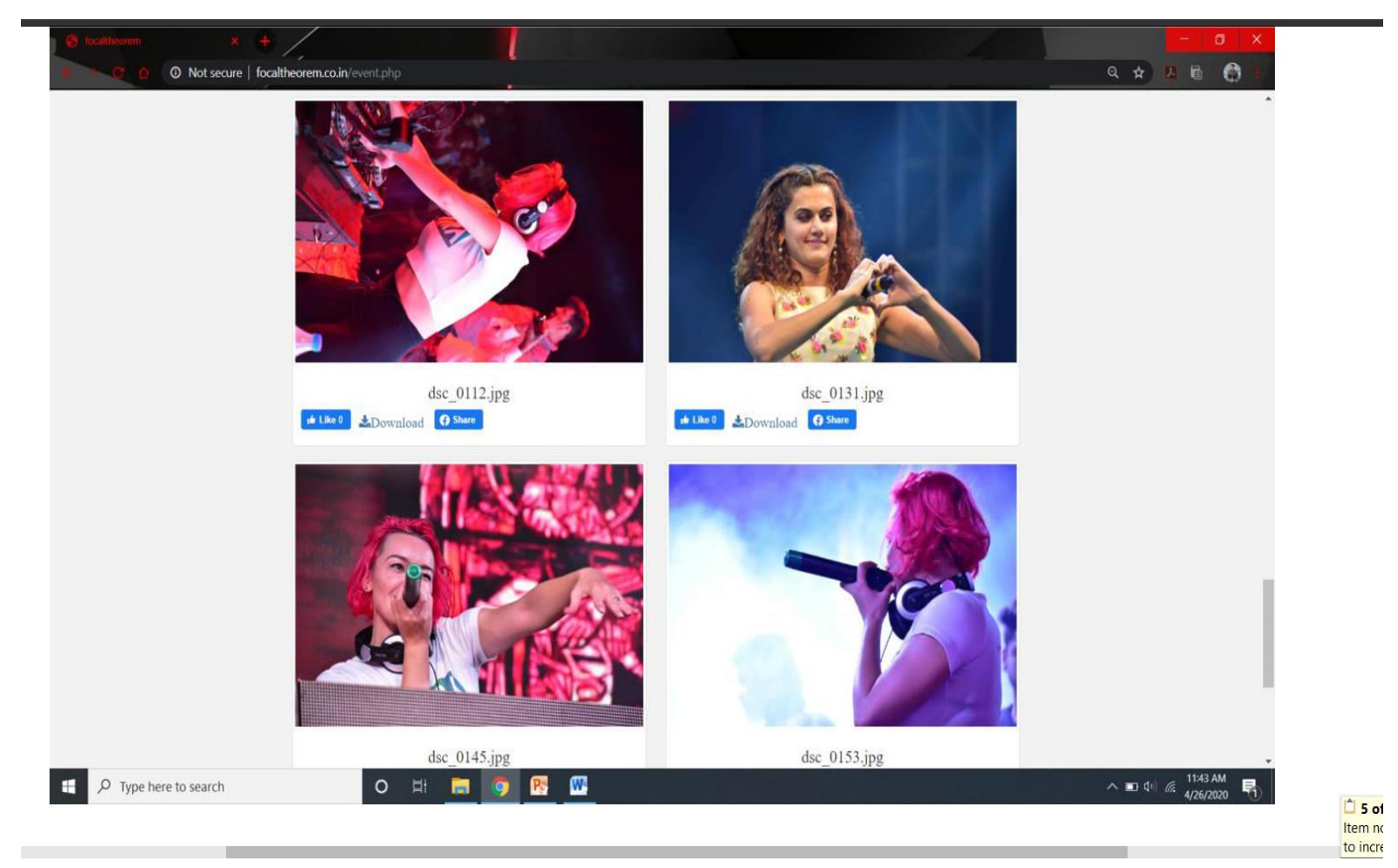

Figure 5: Other pages and Interface 


\section{Conclusion}

In this paper we have preparedthe web applicationusing web development technology to give a platform to share photos, video clipsand stories with others. The projectis developed for both as a final year project to complete the graduation as well as to be used in future by event management groups of college as an official website to share the photos and all the information related the upcoming events like notification, online event registrations and participation as students who want to work in the management field. It is portrayed by

i) an unmistakable division of the duties of the elaborate entertainers (for example understudy, boss, modern contact individual, Users).

ii) a standardizing type of assessment, and

iii) exacting cutoff times for the basic phases of a venture.

One issue that have concerned us over the new years is the manner by which to divide encounters from running the cycle between understudies, chiefs, and Users. This is maybe particularly significant when new and unpracticed understudies are available. This is as yet continuous work.

\section{Acknowledgement}

First of all, we would like to thank Our guide Mr. Himanshu Sharma, and team members Vishesh, Stephen, and Prabhat for meaningful discussions about the contents of this research paper. we will try to make our web App more dynamic and give some extra features so that it become highly responsive as well give the best experience to our users. We want to thank our Supervisor and mentor of the project for guiding us and assisting in our project to help us to make it to final phase.

\section{References}

We have Taken References in our research paper from

B. Olsson, M. Berndtsson, B. Lundell, J. Hansson, 28 May 2015, Running Research-Oriented Final Year Projects for CS and IS Students .

Gates, A., Teller, P. J., Delgado, and Bernat, A.,N. Structuring the student research experience. ACM SIGCSE Bulletin 32, (year-2000).SIGCSE Bulletin 32, 3 (2000), 17-20.- with Success!: A Guide for Computer Science and In-formation Systems Students. Springer-Verlag, 2002.

Boyle, R. D. and Clark, M. A. C., A Personal Theoryof Teaching Computing Through Final Year Projects. CSE 9, March 1999.

For our project we took references from web sites

[1] https://getbootstrap.com/

[2] https://www.geekforgeeks.com

[3] https://www.w3schools.com 